\title{
Transverse Resonance Circuit Modeling and Hertzian Potential Formulation for Integrated Optical Waveguides
}

\author{
Alessandro Massaro*, Roberto Cingolani, Massimo De Vittorio, and Adriana Passaseo ${ }^{\dagger}$ \\ National Nanotechnology Laboratory of CNR-INFM, Distretto Tecnologico-ISUFI, Università del Salento, \\ Via Arnesano, 73100 Lecce, Italy
}

\begin{abstract}
In this work we present a new efficient numerical simulator based on the concepts of transverse resonance and electromagnetic Hertzian potentials. The method includes the transverse resonance circuit modelling (TRCM) well suited with scalar time-domain Hertzian potential modelling (HPM). The transverse circuit provides the effective refractive indices related to the guided modes of an optical waveguide. The simultaneous implementation of the TRCM and HPM approaches provides an accurate time domain electromagnetic field solution for two-dimensional and three-dimensional optical waveguides.
\end{abstract}

Keywords: Optical Circuit Modeling, Transverse Resonance, Scalar Hertzian Potentials, Integrated Optics.

\section{INTRODUCTION}

When considering a dielectric slab we can easily match fields at the interfaces. In the case where our waveguide is a dielectric multilayer structure however, this procedure becomes too involved and inadequate. A different way of looking at the problem, particularly useful in a multilayer situation, is based on network consideration. This is the so called transverse resonance technique ${ }^{1}$ which is represented by thee transverse resonance circuit modeling (TRCM). TRCM provides the effective refractive indices of a dielectric multilayered structure and is well suited with the Hertzian potentials used to solve stratification problems. Hertzian potential method (HPM) represents an accurate and efficient modeling utilized to analyze multilayered dielectric waveguides, ${ }^{2}$ ridge waveguides ${ }^{3}$ and nonlinear optical waveguides, ${ }^{4}$ in a good agreement with experimental results. For a two-dimensional (2-D) problem the proposed time-domain algorithm solves rigorously the electromagnetic field by considering only two scalar equations. For the same problem, the conventional finite difference time domain (FDTD) algorithm solves for three field components which are correlated, by increasing of about $50 \%$ (Ref. [3]) the central processing unit (CPU) time. In a three-dimensional (3-D) case the proposed algorithm solves again two scalar equations instead of six, and subsequently all the electromagnetic (EM) field components

\footnotetext{
*Author to whom correspondence should be addressed.

'Permanent address: IMM-CNR sezione Lecce, University Campus, Lecce-Monteroni 73100, Italy.
}

are obtained by the two scalar potentials as in Hertzian potential formulation. With the introduction of rigorous time domain HPM with the transverse resonance circuit modeling we represent the physical phenomena such as light propagation, scattering and coupling inside optical waveguides by providing a good convergent solution. The simulation algorithm, reported in Figure 1, is based on Hertzian scalar wave equations ${ }^{1-7}$ associated to transmission line circuits including the effective refractive indices evaluation through the TRCM approach. The circuit of the TRCM can be easily matched with the circuital approach of the HPM through the concept of equivalent transmission line. The equivalent transmission lines represent the propagating modes of the optical waveguide. Each propagating mode is solution of the scalar Helmholtz wave equation $^{5-6}$ and is associated to a transmission line with a characteristic impedance which depends on the modal effective refractive index. The second derivatives of the Helmholtz scalar equation introduces an high sensitivity of the numerical error. For this purpose HPM generators ${ }^{2-4}$ are necessary at each dielectric interface in order to model accurately the discontinuous regions of the optical waveguide. These generators allow to model a discontinuous optical waveguide by decreasing the computational cost obtaining good convergent solution. ${ }^{3}$ It is known that the scalar wave equation may lead to inconsistencies because, in inhomogeneous regions such as step discontinuities, it is in general not equivalent to Maxwell's equations. Electromagnetic (EM) scattering problems, including free space, involve the calculation of the fields produced in 


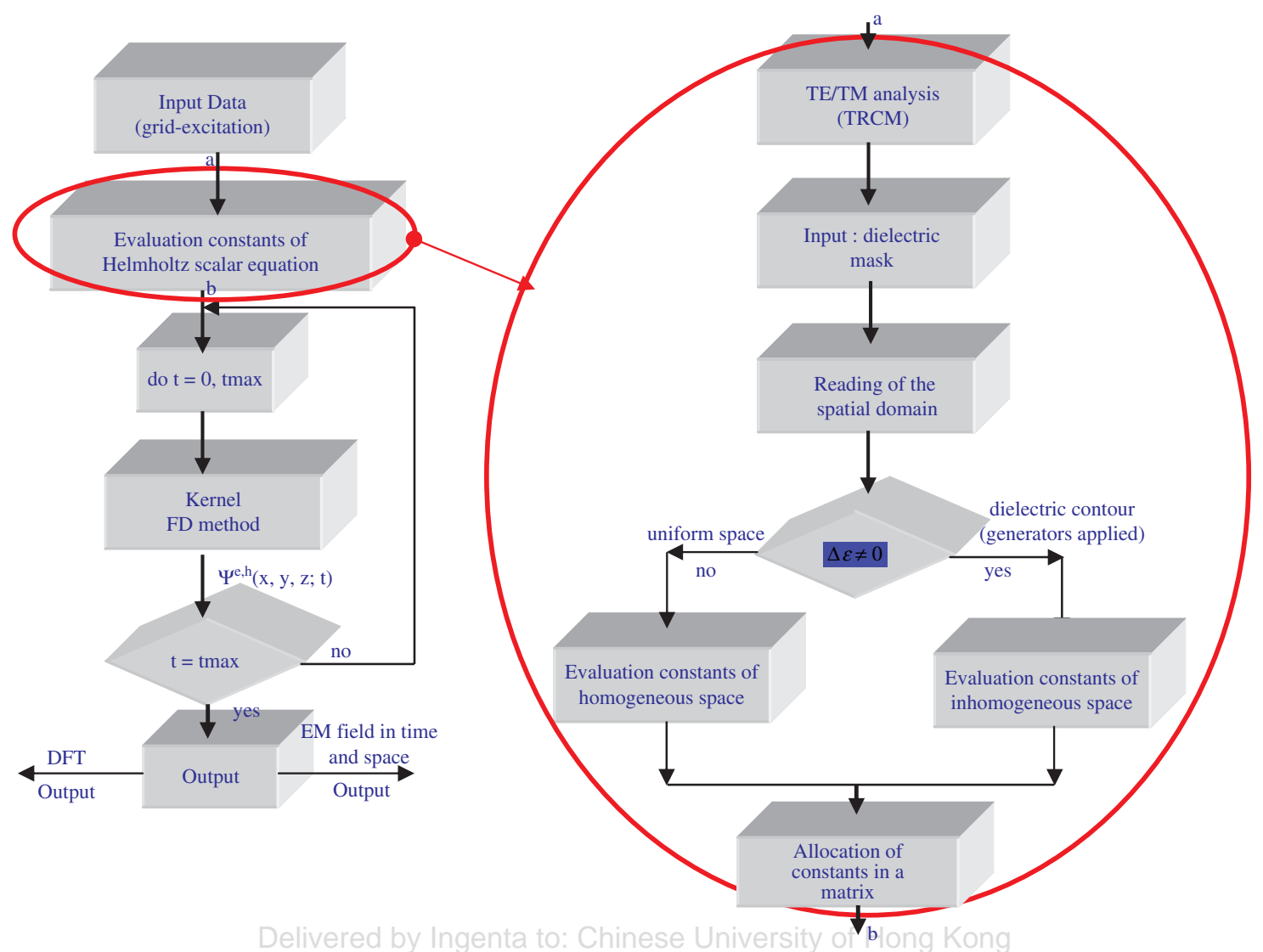

Fig. 1. Finite difference time-domain Hertzian potentials algorithm with transverse resonance circuit modeling.

the presence of these geometrical discontinuities. Such discontinuities may be replaced by equivalent generators, giving an accurate solution of the EM field also for structures with high dielectric contrast. In this way it is also possible to discretise complex dielectric thin multilayer structures by obtaining a closed solution. The numerical approximation introduced by the voltage and current generators of the HPM, combined with the TRCM approach, provides an accurate numerical time-domain tool for the characterization of 2-D and 3-D optical waveguides. In this work we present a complete HPM electromagnetic field formulation implemented with the transverse resonance circuit modeling. We apply the new algorithm to a 3-D tapered ridge waveguide commonly used as integrated optical coupler.

\section{TRANSVERSE RESONANCE CIRCUIT MODELING}

As application of the TRCM method we consider the asymmetrical slab waveguide reported in Figure 2(a). The infinite material cover and substrate are represented by modal admittances. Limited core region is represented by a transmission line. The equivalent modal network considers the total admittance (or impedance) when looking from a reference section (Fig. 2(b)) as the sum of the admittance (or impedance) seen on left and the admittance (or impedance) seen on the right. The resonance condition is obtained by annulling the total admittance (or impedance).

In order to solve the transverse electric (TE) resonance condition it is convenient to normalize the various admittances of Figure 2(b) to $1 / \omega \mu_{0}$, thus obtaining $Y_{01}=k_{\nu}$, $Y_{02}=-j \Delta_{\nu}, Y_{03}=-j \gamma_{\nu}$. By choosing a reference point at $x=d$ we have for TE modes looking towards $x>d$ and $x<d$

$$
\begin{aligned}
\vec{Y}(d)+\overleftarrow{Y}(d) & =Y_{03}+Y_{01} \frac{\left[Y_{02}+j Y_{01} \tan \left(k_{\nu} d\right)\right]}{\left[Y_{01}+j Y_{02} \tan \left(k_{\nu} d\right)\right]} \\
& =-j \gamma_{\nu}+k_{\nu} \frac{\left[-j \Delta_{\nu}+j k_{\nu} \tan \left(k_{\nu} d\right)\right]}{\left[k_{\nu}+j\left(-j \Delta_{\nu}\right) \tan \left(k_{\nu} d\right)\right]} \\
& =0
\end{aligned}
$$

Where $k_{\nu}, \Delta_{\nu}, \gamma_{\nu}$ are the $x$-transverse propagation constants in core, in the substrate and in the cladding region, respectively. We observe that the resonance condition for the transverse magnetic (TM) is obtained by considering the normalization of the admittances to $\omega \varepsilon$, thus obtaining $Y_{01}=1 / k_{\nu}, Y_{02}=-j / \Delta_{\nu}, Y_{03}=-j / \gamma_{\nu}$. In the case of a symmetrical waveguide the circuit can be simplified by considering only half circuit: for symmetry the $E_{y}$ TE field can be either even or odd and so it will present a maximum 
(a)

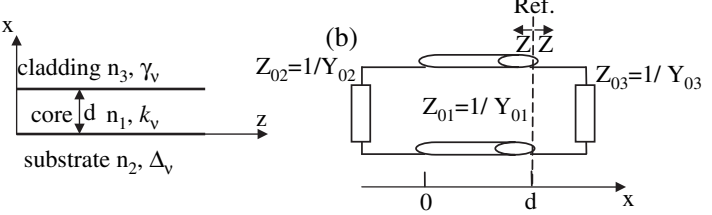

(c)
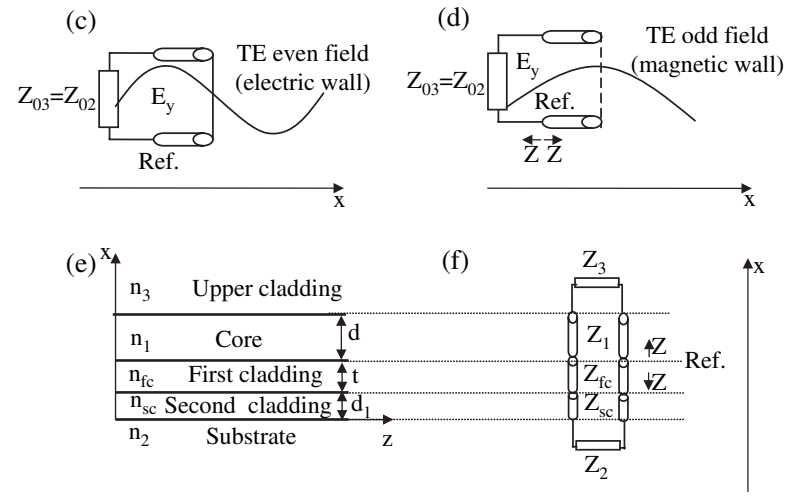

Fig. 2. (a) Asymmetrical slab waveguide. $k_{\nu}, \gamma_{\nu}$ and $\Delta_{\nu}$ represent the propagation constants in the core, in the cladding and in the substrate region, respectively. (b) Transverse equivalent network of TE/TM mode in the asymmetrical slab. (c) Equivalent TE even circuit for a symmetrical waveguide. (d) Equivalent TE odd circuit for symmetrical waveguide. (e) ARROW waveguide. (f) TE/TM transverse equivalent network.

or a minimum in Ref. $=d / 2$ (Ref. and $d$ indicate the reference section and the core thickness respectively in case of TE even mode or TE odd mode as reported in Figs. 2(c) and (d)). This symmetry condition is equivalent to consider an open circuit (magnetic wall of TE odd modes) and a short circuit (electric wall of TE even modes). We observe that in the case of guided modes the core characteristic impedance is real (oscillating field profile), instead the cladding and the substrate admittance are purely imaginary (exponential decay). In the TE even case the transverse resonance condition of a symmetrical waveguide (in which the $x$-transverse air propagation constant is equal to the $x$-transverse substrate propagation constant $\left.\Delta_{\nu}=\gamma_{\nu}\right)$ is given by the total admittance

$$
\begin{aligned}
\vec{Z}+\overleftarrow{Z} & =j Z_{01} \cot \left(k_{\nu}(d / 2)\right)+j Z_{01} \\
& =j \frac{\omega \mu_{0}}{k_{\nu}} \cot \left(k_{\nu}(d / 2)\right)+j \frac{\omega \mu_{0}}{\gamma_{\nu}}=0
\end{aligned}
$$

where the term cotangent represents the input impedance of an open stub circuit (magnetic wall). Regarding the TE odd case the dispersion relation is obtained from the previous formulae by replacing the cotangent functions by tangent. The tangent function represents the input impedance of a short circuit stub (electric wall). The resonance condition can be extended to a generic dielectric multilayer structure. Concerning the ARROW ${ }^{8}$ optical waveguide of Figure 2(e), the TE resonance condition applied to the transverse circuit of Figure 2(f) is

$$
\frac{1}{\gamma_{1}} \cdot \frac{\gamma_{1}+\gamma_{3} \tanh \left(\gamma_{1} d\right)}{\gamma_{3}+\gamma_{1} \tanh \left(\gamma_{1} d\right)}+\frac{1}{\gamma_{\mathrm{fc}}} \cdot \frac{\gamma_{\mathrm{fc}}+\bar{\gamma}_{\mathrm{sc}} \tanh \left(\gamma_{\mathrm{fc}} t\right)}{\bar{\gamma}_{\mathrm{sc}}+\gamma_{\mathrm{fc}} \tanh \left(\gamma_{\mathrm{fc}} t\right)}=0
$$

with

$$
\bar{\gamma}_{\mathrm{sc}}=\frac{1}{\gamma_{\mathrm{sc}}} \cdot \frac{\gamma_{\mathrm{sc}}+\gamma_{2} \tanh \left(\gamma_{\mathrm{sc}} d_{1}\right)}{\gamma_{2}+\gamma_{\mathrm{sc}} \tanh \left(\gamma_{\mathrm{sc}} d_{1}\right)}
$$

where $\gamma_{3}, \gamma_{1}, \gamma_{\mathrm{fc}}, \gamma_{\mathrm{sc}}, \gamma_{2}$ are the propagation constants in the upper cladding, in the core, in the first cladding, in the second cladding and in the substrate region, respectively.

\section{HERTZIAN POTENTIALS FORMULATION AND MODELING}

The Hertzian electric and magnetic vectors ${ }^{5-7}$ are represented in rectangular coordinates by

$$
\begin{aligned}
& \bar{\Pi}_{e}=\mathbf{a} \psi^{e}(x, y, z, t) \\
& \bar{\Pi}_{h}=\mathbf{a} \psi^{h}(x, y, z, t)
\end{aligned}
$$

where a is unit vector, $\psi^{e} \psi^{h}$ represent the TE and the TM polarization, respectively. From (1) it is possible to evaluate all the components of the electromagnetic (EM) field as

$$
\begin{aligned}
& \overline{\mathbf{E}}=\nabla \nabla \cdot \bar{\Pi}_{e}-\varepsilon \mu \frac{\partial^{2}}{\partial t^{2}} \bar{\Pi}_{e}-\mu \frac{\partial}{\partial t}\left(\nabla \times \bar{\Pi}_{h}\right) \\
& \overline{\mathbf{H}}=\nabla \nabla \cdot \bar{\Pi}_{h}-\varepsilon \mu \frac{\partial^{2}}{\partial t^{2}} \bar{\Pi}_{h}+\varepsilon \frac{\partial}{\partial t}\left(\nabla \times \bar{\Pi}_{e}\right)
\end{aligned}
$$

where

$$
\begin{aligned}
\nabla \cdot \bar{\Pi}_{e, h}= & \partial_{x} \psi^{e, h}+\partial_{y} \psi^{e, h}+\partial_{z} \psi^{e, h} \\
\nabla \nabla \cdot \bar{\Pi}_{e, h}^{1}= & \hat{x} A^{e, h}+\hat{y} B^{e, h}+\hat{z} C^{e, h} \\
\nabla \times \bar{\Pi}_{e, h}= & \hat{x}\left(\partial_{y} \psi^{e, h}-\partial_{z} \psi^{e, h}\right)+\hat{y}\left(\partial_{z} \psi^{e, h}-\partial_{x} \psi^{e, h}\right) \\
& +\hat{z}\left(\partial_{x} \psi^{e, h}-\partial_{y} \psi^{e, h}\right)
\end{aligned}
$$

with

$$
\begin{aligned}
& A^{e, h}=\partial_{x}^{2} \psi^{e, h}+\partial_{y x} \psi^{e, h}+\partial_{z x} \psi^{e, h} \\
& B^{e, h}=\partial_{x y} \psi^{e, h}+\partial_{y}^{2} \psi^{e, h}+\partial_{z y} \psi^{e, h} \\
& C^{e, h}=\partial_{x z} \psi^{e, h}+\partial_{y z} \psi^{e, h}+\partial_{z}^{2} \psi^{e, h}
\end{aligned}
$$

By using (7), (8) the Eq. (6) becomes

$$
\begin{gathered}
\overline{\mathbf{E}}=\hat{x} A^{e}+\hat{y} B^{e}+\hat{z} C^{e}-\hat{x} \mu \varepsilon \partial_{t}^{2} \psi^{e}-\hat{y} \mu \varepsilon \partial_{t}^{2} \psi^{e}-\hat{z} \mu \varepsilon \partial_{t}^{2} \psi^{e} \\
-\mu \partial_{t}\left(\hat{x}\left(\partial_{y} \psi^{h}-\partial_{z} \psi^{h}\right)+\hat{y}\left(\partial_{z} \psi^{h}-\partial_{x} \psi^{h}\right)\right. \\
\left.+\hat{z}\left(\partial_{x} \psi^{h}-\partial_{y} \psi^{h}\right)\right) \\
\overline{\mathbf{H}}=\hat{x} A^{h}+\hat{y} B^{h}+\hat{z} C^{h}-\hat{x} \mu \varepsilon \partial_{t}^{2} \psi^{h}-\hat{y} \mu \varepsilon \partial_{t}^{2} \psi^{h} \\
-\hat{z} \mu \varepsilon \partial_{t}^{2} \psi^{h}+\varepsilon \partial_{t}\left(\hat{x}\left(\partial_{y} \psi^{e}-\partial_{z} \psi^{e}\right)\right. \\
\left.+\hat{y}\left(\partial_{z} \psi^{e}-\partial_{x} \psi^{e}\right)+\hat{z}\left(\partial_{x} \psi^{e}-\partial_{y} \psi^{e}\right)\right)
\end{gathered}
$$

All the EM component of Eq. (9) for a general unit vector a are given by

$$
\begin{aligned}
& E_{x}=A^{e}-\mu \varepsilon \partial_{t}^{2} \psi^{e}+\mu \partial_{t} \partial_{z} \psi^{h}-\mu \partial_{t} \partial_{y} \psi^{h} \\
& E_{y}=B^{e}-\mu \varepsilon \partial_{t}^{2} \psi^{e}-\mu \partial_{t} \partial_{z} \psi^{h}+\mu \partial_{t} \partial_{x} \psi^{h} \\
& E_{z}=C^{e}-\mu \varepsilon \partial_{t}^{2} \psi^{e}-\mu \partial_{t} \partial_{x} \psi^{h}+\mu \partial_{t} \partial_{y} \psi^{h} \\
& H_{x}=A^{h}-\mu \varepsilon \partial_{t}^{2} \psi^{h}+\varepsilon \partial_{t} \partial_{y} \psi^{e}-\varepsilon \partial_{t} \partial_{z} \psi^{e}
\end{aligned}
$$




$$
\begin{aligned}
& H_{y}=B^{h}-\varepsilon \mu \partial_{t}^{2} \psi^{h}+\varepsilon \partial_{t} \partial_{z} \psi^{e}-\varepsilon \partial_{t} \partial_{x} \psi^{e} \\
& H_{z}=C^{h}-\mu \varepsilon \partial_{t}^{2} \psi^{h}+\varepsilon \partial_{t} \partial_{x} \psi^{e}-\varepsilon \partial_{t} \partial_{y} \psi^{e}
\end{aligned}
$$

The potentials $\psi^{e, h}(x, y, z, t)$ represents the solutions of the homogeneous wave equations for a non-dissipative medium

$$
\nabla^{2} \psi^{e, h}(x, y, z, t)-\mu \varepsilon \frac{\partial^{2} \psi^{e, h}(x, y, z, t)}{\partial t^{2}}=0
$$

and for a dissipative medium

$$
\begin{gathered}
\nabla^{2} \psi^{e, h}(x, y, z, t)-\mu \varepsilon \frac{\partial^{2} \psi^{e, h}(x, y, z, t)}{\partial t^{2}} \\
-\mu \sigma \frac{\partial \psi^{e, h}(x, y, z, t)}{\partial t}=0
\end{gathered}
$$

where $\varepsilon$ is the electrical permittivity (farads/meter), $\mu$ is magnetic permeability (henrys/meter), and $\sigma$ is the electric conductivity (siemens/meter) which is zero in a perfect dielectric. For a 2-D optical device the HPM algorithm, shown in Figure 1, solves Eqs. (11) and (12) by considering the equivalent effective TE/TM indices found through the TRCM approach ( $\varepsilon$ is substituted with the effective index $\left.\varepsilon_{\text {eff }}\right)$. For this purpose the HPM algorithm is perfectly matched with TRCM approach: $\psi^{e}$ and $\psi^{h}$ represent two guided modes characterized effective permittivity index $\varepsilon_{\text {eff }}$ evaluated by the TRCM approach. Each mode propagates in the optical waveguide as a signal which travels in a transmission line characterized by a characteristic impedance

$$
Z_{e, h}=\frac{1}{\sqrt{\varepsilon_{\text {eff }_{e, h}}}} \sqrt{\frac{\mu_{0}}{\varepsilon_{0}}}
$$

By analyzing the asymmetrical slab waveguide, the HPM algorithm of Figure 1 evaluates before the kernel iterations the TE and the TM equivalent effective refractive indices. Figure 3 shows the calculated TE/TM effective refractive indices by varying the core thickness for a slab waveguide with $n_{1}(\mathrm{GaAs})=3.408, n_{2}(\mathrm{AlGaAs})=3.042, n_{3}=1$ and for a working wavelength of $\lambda_{0}=1.31 \mu \mathrm{m}$. A practical application of the asymmetrical slab waveguide is reported in Figure 4(a) where a grating at the interface between the

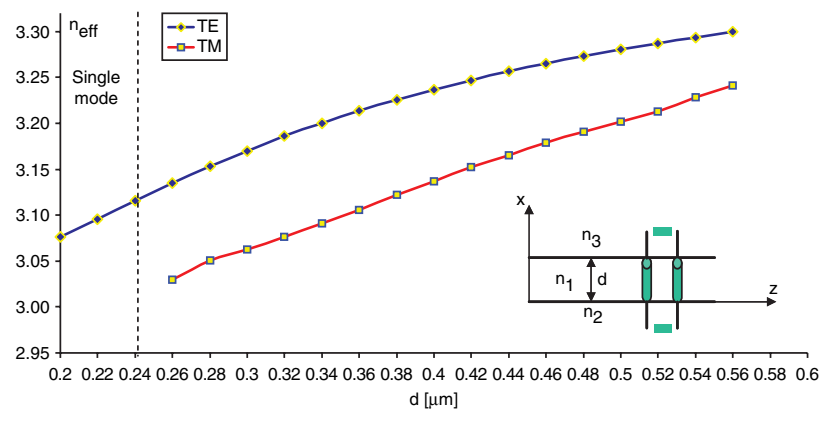

Fig. 3. TRCM approach: TE and TM effective refractive indices for an asymmetrical slab waveguide for different values of the core thickness $d$. Inset: equivalent transverse circuit. (a)

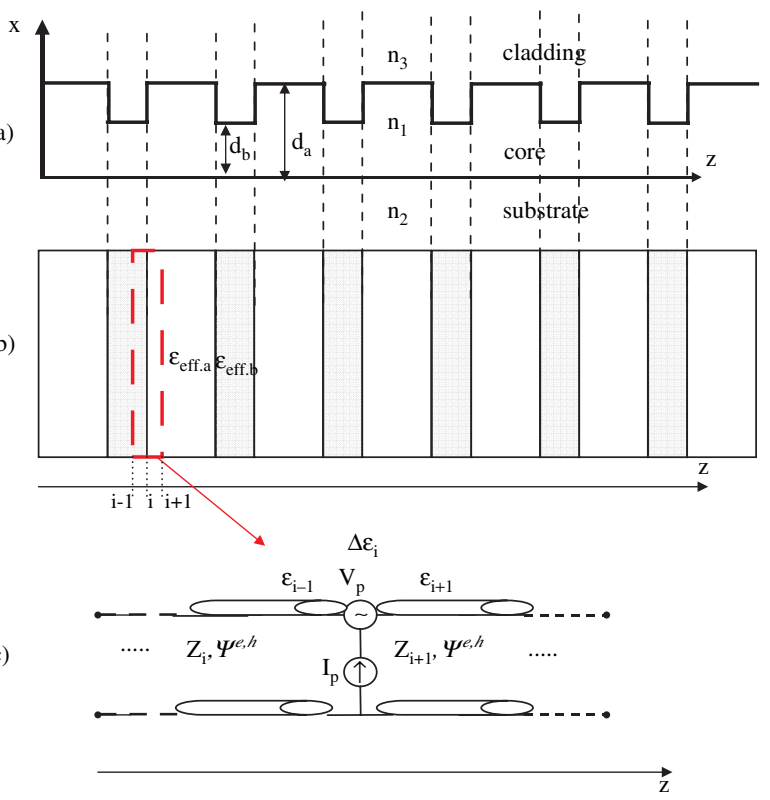

Fig. 4. (a) Asymmetrical slab waveguide with grating. (b) Equivalent dielectric structure. (c) Equivalent generator HPM modeling at the dielectric interface.

core and the cladding region is considered. In this case the HPM algorithm evaluates the effective refractive indices for the thicknesses $d_{a}$ and $d_{b}$ reported in Figure 4(a) by obtaining the equivalent multilayered dielectric structure illustrated in Figure 4(b). In order to the reduce the numerical error of the temporal second derivatives of Eqs. (11) and (12), the HPM generators $V_{P}$ and $I_{P}$ (see Fig. 4(c)) are placed directly on the dielectric interface nodes. The efficacy of the HPM generators is shown in Figure 5, where is reported the time evolution of the $E_{y}$ component (normal to plane of the figure) one cell before the dielectric multilayered structure of Figure 4(b). The generators in

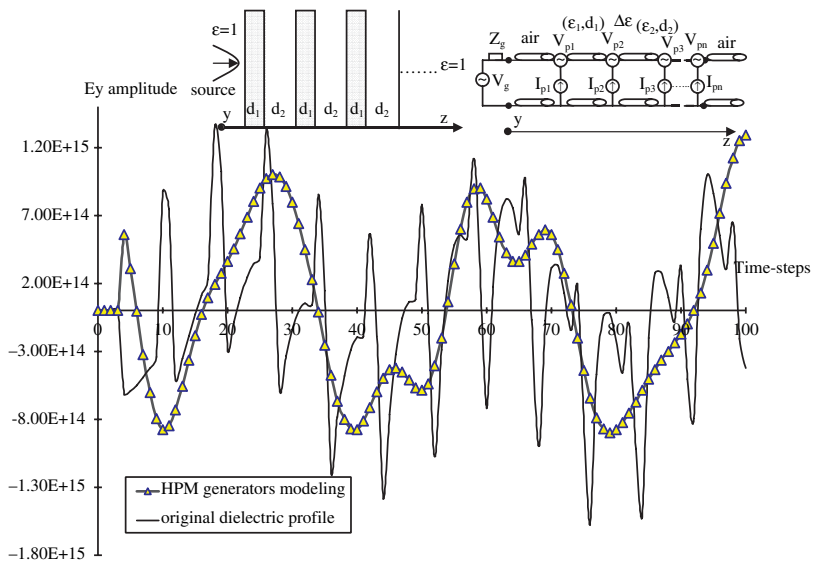

Fig. 5. HPM generators modeling: convergent solution of the TE $E_{y}$ component regarding the periodical waveguide of Figure 4(a) with $d_{a}=$ $0.35 \mu \mathrm{m}, d_{b}=0.25 \mu \mathrm{m}, n_{1}(\mathrm{GaAs})=3.408, n_{2}(\mathrm{AlGaAs})=3.042, n_{3}=1$ and $d 1=d 2=0.3 \mu \mathrm{m}$. Inset: equivalent multilayered structure and equivalent HPM circuit model with generators. 
this case provides a no-oscillating closed solution also by considering low grid sizes. The $E_{y}$ oscillations, obtained by considering the original dielectric profile without HPM generators, represent the numerical errors. Now we quantify $V_{p}$ and $I_{p}$ by a finite difference ${ }^{9}$ parametric evaluation of Eq. (11). The scalar wave Eq. (11) for a non-dissipative medium can be rewritten as

$$
\begin{gathered}
\nabla^{2} \psi_{e, h}(x, y, z, t)-\mu \varepsilon \frac{\partial^{2} \psi_{e, h}(x, y, z, t)}{\partial t^{2}} \\
-\mu \frac{\partial^{2} P_{\text {pert }}(x, y, z, t)}{\partial t^{2}}=0
\end{gathered}
$$

where

$$
P_{\text {pert }}(x, y, z, t)=\Delta \varepsilon(x, y, z, t) \psi_{e, h}(x, y, z, t)
$$

represents the dielectric polarization which takes into account the dielectric discontinuity. For a 3-D case we have:

$$
\begin{array}{ll}
\Delta \varepsilon=\varepsilon_{i+1}-\varepsilon_{i} & i=\text { cell position in } x \text { direction } \\
\Delta \varepsilon=\varepsilon_{j+1}-\varepsilon_{j} & j=\text { cell position in } y \text { direction } \\
\Delta \varepsilon=\varepsilon_{k+1}-\varepsilon_{k} & k=\text { cell position in } z \text { direction }
\end{array}
$$

Therefore we solve Eq. (14) in proximity of the dielectric interfaces, and Eq. (11) in the homogenous regions. The difference between the parametric solution of Eq. (14), and Eq. (11) in the iterative form, is in the coefficients; in fact for one propagation direction Eqs. (11) and (14) become respectively

$$
\begin{gathered}
\psi^{n+1}(j)\left(\frac{\mu \varepsilon}{(\Delta t)^{2}}\right) \\
=\psi^{n}(j+1)\left(\frac{1}{(\Delta z)^{2}}\right)+\psi^{n}(j)\left(\frac{2 \mu \varepsilon}{(\Delta t)^{2}}-\frac{2}{(\Delta z)^{2}}\right) \\
\quad+\psi^{n-1}(j)\left(-\frac{\mu \varepsilon}{(\Delta t)^{2}}\right)+\psi^{n}(j-1)\left(\frac{1}{(\Delta z)^{2}}\right) \\
\psi^{n+1}(j)\left(\frac{\mu \varepsilon}{(\Delta t)^{2}}+\frac{\mu \Delta \varepsilon}{(\Delta t)^{2}}\right) \\
=\psi^{n}(j+1)\left(\frac{1}{(\Delta z)^{2}}\right)+\psi^{n}(j)\left(\frac{2 \mu \varepsilon}{(\Delta t)^{2}}-\frac{2}{(\Delta z)^{2}}+\frac{2 \mu \Delta \varepsilon}{(\Delta t)^{2}}\right) \\
+\psi^{n-1}(j)\left(-\frac{\mu \varepsilon}{(\Delta t)^{2}}-\frac{\mu \Delta \varepsilon}{(\Delta t)^{2}}\right)+\psi^{n}(j-1)\left(\frac{1}{(\Delta z)^{2}}\right)(20)
\end{gathered}
$$

where $n$ and $j$ indicate the time step and the position along one direction (in this case $z$-direction), respectively. By comparing the implemented formula

$$
\begin{aligned}
\psi^{n+1}(j)= & \psi^{n}(j+1)\left(\frac{b}{a}\right)+\psi^{n}(j)\left(\frac{2 a-2 b}{a}\right) \\
& +\psi^{n-1}(j)(-1)+\psi^{n}(j-1)\left(\frac{b}{a}\right)
\end{aligned}
$$

$$
\begin{aligned}
\psi^{n+1}(j)= & \psi^{n}(j+1)\left(\frac{b}{a^{\prime}}\right)+\psi^{n}(j)\left(\frac{2 a^{\prime}-2 b}{a^{\prime}}\right) \\
& +\psi^{n-1}(j)(-1)+\psi^{n}(j-1)\left(\frac{b}{a^{\prime}}\right)
\end{aligned}
$$

with

$$
\begin{aligned}
a & =\frac{\mu \varepsilon}{(\Delta t)^{2}} \\
a^{\prime} & =a+\frac{\mu \Delta \varepsilon}{(\Delta t)^{2}} \\
b & =\frac{1}{(\Delta z)^{2}}
\end{aligned}
$$

we found the effect of the generators $V_{p}$ and $I_{p}$.

\section{3-D OPTICAL WAVEGUIDES}

By applying the TRCM approach it is possible to analyze complex 3-D optical waveguides in a 2-D case. As example we consider the 3-D tapered ridge waveguide of Figure 6(a): this waveguide can be analyzed as a 2D waveguide by evaluating the effective refractive index $n_{\text {effy }}$ in the $y$-direction. By considering the cross section of the tapered ridge waveguide at $z=0$ we define the two region reported in Figure 6(b). The TRCM method evaluates through the resonance circuit model the effective refractive index $n_{\text {effyl }}$ in the region 1 (referred to the waveguide with core thickness $d$ ), and the index $n_{\text {effy } 1}$ in the region 2 (referred to the waveguide with core thickness $D$ ). In Figures 6(c) and (d) we show the HPM equivalent circuit before the tapered region and on the slanted profile, respectively. The complete modal classification ${ }^{1}$ is given by applying again the TRCM also along the $x$-direction.
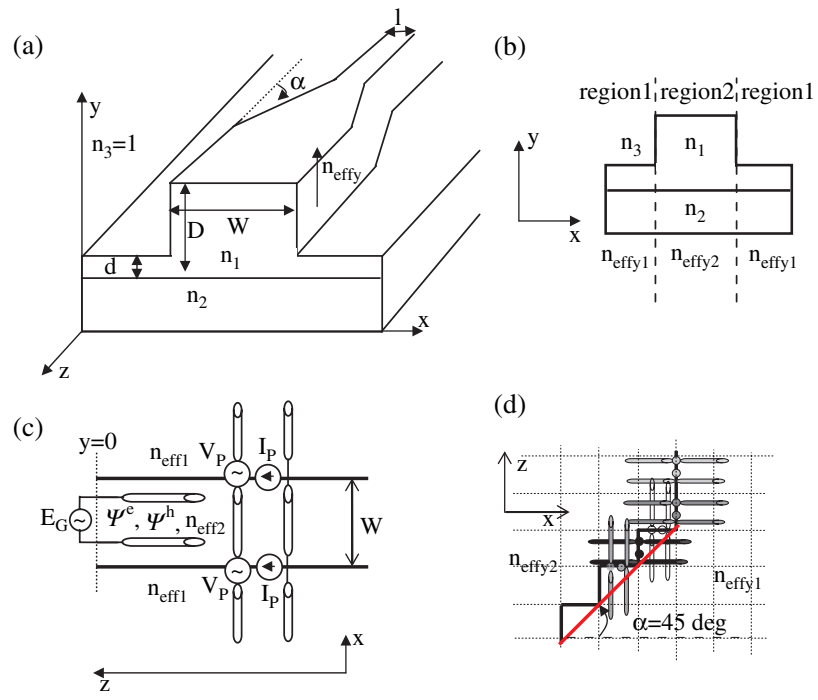

(d)

Fig. 6. (a) 3D tapered ridge waveguide. (b) Cross section at $z=0$ and effective refractive indices. (c) HPM equivalent circuit before tapered region. (d) HPM circuit model with generators on the slanted profile. 


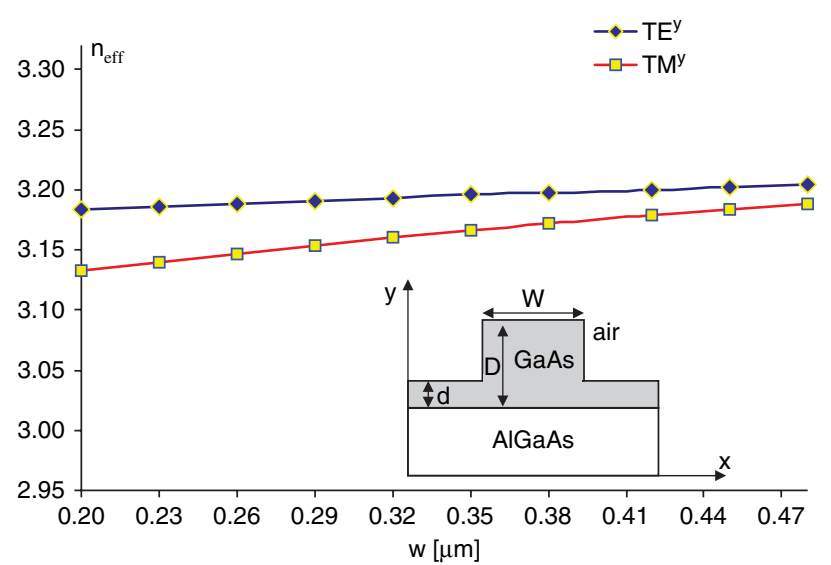

Fig. 7. $\mathrm{TE}^{y}$ and the $\mathrm{TM}^{y}$ modes for different widths $W$ of a ridge waveguide with $n_{1}(\mathrm{GaAs})=3.408, n_{2}(\mathrm{AlGaAs})=3.042, n_{3}=1, d=$ $0.3 \mu \mathrm{m}$, and $D=0.54 \mu \mathrm{m}$. The used working wavelength is $\lambda_{0}=$ $1.31 \mu \mathrm{m}$.

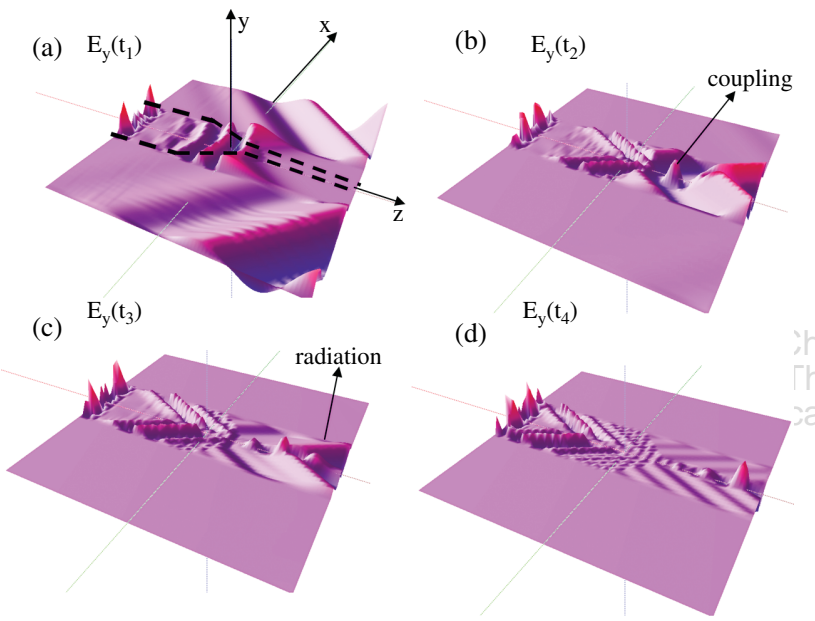

Fig. 8. Time evolution of the $E_{y}$ component after (a) $t_{1}$, (b) $t_{2}$, (c) $t_{3}$, and (d) $t_{4}$ time-step with $t_{1}<t_{2}<t_{3}<t_{4}$. The simulated waveguide is characterized by $n_{1}(\mathrm{GaAs})=3.408, n_{2}(\mathrm{AlGaAs})=3.042, n_{3}=1, d=$ $0.3 \mu \mathrm{m}, D=0.54 \mu \mathrm{m}, W=10 \mu \mathrm{m}, l=0.5 \mu \mathrm{m}$, and $\alpha=45^{\circ}$. The used source is a carrier at $\lambda_{0}=1.31 \mu \mathrm{m}$ modulated by an exponential signal.

In particular the $\mathrm{TE}^{y}$ is defined by the components $E_{x}$, $E_{z}, H_{x}, H_{y}, H_{z}$, with $E_{y}=0$, and the $\mathrm{TM}^{y}$ is represented by the components $H_{x}, H_{z}, E_{x}, E_{y}, E_{z}$, with $H_{y}=0$. The $\mathrm{TE}^{y}$ and the $\mathrm{TM}^{y}$ modes of a ridge waveguide with
$n_{1}(\mathrm{GaAs})=3.408, n_{2}(\mathrm{AlGaAs})=3.042, n_{3}=1, d=$ $0.3 \mu \mathrm{m}$, and $D=0.54 \mu \mathrm{m}$ are reported in Figure 7. In this case we vary the ridge width $\mathrm{W}$ by fixing the working wavelength at $\lambda_{0}=1.31 \mu \mathrm{m}$.

Moreover coupling and the radiation aspects are highlighted by Figure 8 which illustrates the time evolution of the $E_{y}$ component after different time-steps. In this simulation we consider as source a carrier at $\lambda_{0}=1.31 \mu \mathrm{m}$ modulated by an exponential signal and the absorbing boundary conditions (ABCs).$^{10}$ In this case extremely small boundary reflection coefficient of the order of $10^{-11}$ is obtained in the simulations by enhancing the accuracy of the solution for long simulation time steps.

\section{CONCLUSION}

In this work we analyze an algorithm totally based on equivalent circuits. The TRCM circuit characterizes all the propagating modes of an optical waveguide, and the HPM generators modelling provides a closed solution of the electromagnetic field. The TRCM approach combined with the HPM formulation represents a new tool for the design of 2-D and 3-D multilayered structures.

\section{References}

1. T. Rozzi and M. Mongiardo, Open Electromagnetic Waveguides, IEE Electromagnetic Waves Series 43, London (1997), pp. 21-50.

2. A. Massaro and T. Rozzi, Opt. Express 14, 2027 (2006).

3. A. Massaro, M. Grande, R. Cingolani, A. Passaseo, and M. De Vittorio, Opt. Express 15, 16484 (2007).

4. A. Massaro, V. Tasco, M. T. Todaro, R. Cingolani, M. De Vittorio, and A. Passaseo, Opt. Express 16, 14496 (2008).

5. T. Rozzi and M. Farina, Advanced Electromagnetic Analysis of Passive and Active Planar Structures, IEE Electromagnetic Wave Series 46, London (1999).

6. C. G. Someda, Onde Elettromagnetiche, UTET, Torino (1996).

7. M. Couture, IEEE Trans. Microwave Theory and Tech. 35, 288 (1987).

8. L. Pierantoni, A. Massaro, and T. Rozzi, IEEE Trans. Microw. Theory Tech. 53, 1856 (2005).

9. A. Taflove and S. C. Hagness, Computational Electrodynamic: The Finite-Difference Time-Domain Method, Arthec House Publishers, Sec. ed., London (2000).

10. G. Mur, IEEE Trans. Electromagnetic Compatibility 23, 377 (1981).

Received: 30 October 2008. Accepted: 18 November 2008. 